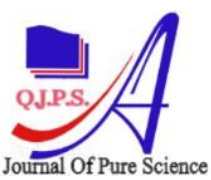

Received : 23/12/2019

\section{Al-Qadisiyah Journal Of Pure Science (QJPS) \\ Vol. 25, No. 1,math pp. 11-15, Year2020}

\title{
ON THE STABILITY CONDITIONS OF 2D TIME FRACTIONAL DIFFUSION EQUATION
}

\author{
Esraa Abbas Al-taai a and Adel Rashed A. Ali b,* \\ ${ }^{\text {a } U n i v e r s i t y ~ o f ~ B a g h d a d, ~ B a g h d a d, ~ I r a q, ~ E m a i l ~(e s r a a a b a s 193 @ y a h o o . c o m) ~}$ \\ ${ }^{\mathrm{b}}$ Department of Mathematics, College of Education for Pure Science (Ibn al-Haitham), University \\ of Baghdad, Baghdad, Iraq, Email (adil.r.a@ihcoedu.uobaghdad.edu.iq)
}

\begin{abstract}
:
The Caputo definition of fractional derivative has been used for the time derivative for the two-dimensional time-fractional diffusion equation. The stability condition obtained by reformulation the classical multilevel technique on the finite difference scheme. A numerical example gives a good agreement with the theoretical result
\end{abstract}

KEYWORDS: Fractional Time Diffusion Equation, Caputo Fractional Derivative, Finite difference, Multilevel, Stability Analysis. 


\section{Introduction}

In the last decades, there was huge attention to deal with various problems employing fractional calculus. The Fractional parabolic partial differential equation used with many problems in physics, mechanics and dynamical systems and many physical processes. These problems will be more accurate and precise if it describes by fractional-order derivative (Miller 1993, Podlubny 1999). The computational of problems with fractional derivative are costs too much comparing with the case of integer derivative due to memory and hereditary properties of a fractional derivative but the fractional order derivatives provide useful tools for the description of memory and hereditary properties of different substances (Herrmann 2011). the fundamental background of fractional calculus can be found in (Herrmann 2011, Miller 1993, Podlubny 1999).

Consider the diffusion in stranded form

$\frac{\partial T(x, y, t)}{\partial t}=\frac{\partial^{2} T(x, y, t)}{\partial x^{2}}+\frac{\partial^{2} T(x, y, t)}{\partial y^{2}}+Q(x, y, t)$

with the initial and boundary conditions are given as follow:

$$
\begin{aligned}
& T(x, y, 0)=\varphi(x, y) ; \quad(x, y) \in \Omega \\
& \left.T(x, y, t)\right|_{\partial \Omega}=0 ; 0 \leq t
\end{aligned}
$$

Where $\Omega=\{(x, y) \mid 0 \leq x \leq 1,0 \leq y \leq 1\}$. The time fractional diffusion equation is obtained from the standard diffusion equation by replacing the first order time derivative with a fractional derivative of order $\alpha$, where $0<\alpha<1$, so the fractional time diffusion equation can be written as

$\frac{\partial^{\alpha} T(x, y, t)}{\partial t^{\alpha}}=\frac{\partial^{2} T(x, y, t)}{\partial x^{2}}+\frac{\partial^{2} T(x, y, t)}{\partial y^{2}}+Q(x, y, t)$

with the same initial and boundary conditions of equation (1). Our main task is to obtain the stability conditions of the explicit finite difference scheme of the fractional time diffusion equation where the fractional order time derivative is the Caputo fractional derivative of order $\alpha$ defined by (Miller 1993, Podlubny 1999):

$\frac{\partial^{\alpha} T(x, y, t)}{\partial t^{\alpha}}=\frac{1}{\Gamma(1-\alpha)} \int_{0}^{t}(t-s)^{-\alpha} \frac{\partial T(x, y, s)}{\partial s} d s$

The time derivative in the diffusion equation is of the first order, so most of the famous schemes are uses the forward or the backward finite difference formula and replaced with the time derivative in the diffusion equation to get two-level time scheme, then Von Neumann technique for stability can be used because its technique is for two-level scheme. But with the time-fractional order derivative, the scheme will be a multilevel scheme because of the memory and hereditary properties of fractional order derivative and the Von Neumann technique can not be used to find the stability conditions.

In this article, we use a reformulation of the classical multilevel technique and produce a simple and easy method to find the stability conditions for the time-fractional diffusion equation in two dimensions.

\section{The Explicit Finite Difference Scheme}

For $i=0,1,2, \cdots, M, j=0,1,2, \cdots, N, k=0,1,2, \cdots, n$ define $x_{i}=i \Delta x, y_{j}=j \Delta y, t_{k}=k \tau$ and $T_{i, j}^{k}, Q_{i, j}^{k}$ be the numerical approximations to $T\left(x_{i}, y_{j}, t_{k}\right)$ and $Q\left(x_{i}, y_{j}, t_{k}\right)$. The time fractional derivative can be express by the following scheme (for more details see (Zhuang 2007)):

$\frac{\partial^{\alpha} T\left(x_{i}, y_{j}, t_{n+1}\right)}{\partial t^{\alpha}}=w_{\alpha}^{\tau} \sum_{k=0}^{n} b_{k}^{\alpha}\left(T_{i, j}^{n-k+1}-T_{i, j}^{n-k}\right)$

where $w_{\alpha}^{\tau}=\frac{\tau^{-\alpha}}{\Gamma(2-\alpha)}$ and $b_{k}^{\alpha}=(k+1)^{1-\alpha}-k^{1-\alpha}$, for $k=$ $0,1,2, \cdots, n$. It is easy to verify that $b_{0}=1, b_{k}>b_{k+1}$. The equation (4) can be rearranged in the form

$$
\frac{\partial^{\alpha} T\left(x_{i}, y_{j}, t_{n+1}\right)}{\partial t^{\alpha}}=w_{\alpha}^{\tau}\left[T_{i, j}^{n+1}-\sum_{k=1}^{n} c_{k}^{\alpha} T_{i, j}^{n-k+1}-b_{n}^{\alpha} T_{i, j}^{0}\right]
$$

where $c_{k}^{\alpha}=b_{k}^{\alpha}-b_{k+1}^{\alpha}$. Replacing the time derivative in equation (2) using equation (5) at the grid point $\left(x_{i}, y_{j}, t_{n}\right)$ and the space derivatives with the central difference approximation at the same grid point $\left(x_{i}, y_{j}, t_{n}\right)$, then the explicit scheme have the following difference equation

$$
\begin{array}{r}
w_{\alpha}^{\tau}\left[T_{i, j}^{n+1}-\sum_{k=1}^{n} c_{k}^{\alpha} T_{i, j}^{n-k+1}-b_{n}^{\alpha} T_{i, j}^{0}\right]=\frac{1}{(\Delta x)^{2}} \delta_{x}^{2} T_{i, j}^{n} \\
+\frac{1}{(\Delta y)^{2}} \delta_{y}^{2} T_{i, j}^{n}+Q_{i, j}^{n}
\end{array}
$$

where $Q_{i, j}^{n}=Q(i \Delta x, j \Delta y, n \Delta t), \delta_{x}^{2} T_{i, j}^{n}=T_{i-1, j}^{n}-2 T_{i, j}^{n}+T_{i+1, j}^{n}$ and $\delta_{y}^{2} T_{i, j}^{n}=T_{i, j-1}^{n}-2 T_{i, j}^{n}+T_{i, j+1}^{n}$. For simplicity let $\Delta x=$ $\Delta y=h$ then

$$
\begin{gathered}
\begin{array}{c}
w_{\alpha}^{\tau}\left[T_{i, j}^{n+1}-\sum_{k=1}^{n} c_{k}^{\alpha} T_{i, j}^{n-k+1}-b_{n}^{\alpha} T_{i, j}^{0}\right]= \\
+Q_{i, j}^{n}\left[\left(\delta_{x}^{2}+\delta_{y}^{2}\right) T_{i, j}^{n}\right] \\
\text { or } T_{i, j}^{n+1}-\sum_{k=1}^{n} c_{k}^{\alpha} T_{i, j}^{n-k+1}-b_{n}^{\alpha} T_{i, j}^{0}= \\
r_{\alpha}\left[\Gamma(2-\alpha)\left(\delta_{x}^{2}+\delta_{y}^{2}\right) T_{i, j}^{n}\right]+\frac{1}{w_{\alpha}^{\tau}} Q_{i, j}^{n}
\end{array}
\end{gathered}
$$

where $r_{\alpha}=\frac{\tau^{\alpha}}{h^{2}}$, and this can be rewritten as

$$
\begin{aligned}
T_{i, j}^{n+1}=\sum_{k=1}^{n} & c_{k}^{\alpha} T_{i, j}^{n-k+1}+b_{n}^{\alpha} T_{i, j}^{0} \\
& +r_{\alpha} \Gamma(2-\alpha)\left[\left(\delta_{x}^{2}+\delta_{y}^{2}\right) T_{i, j}^{n}\right]+\frac{1}{w_{\alpha}^{\tau}} Q_{i, j}^{n}
\end{aligned}
$$

\section{The Multilevel Technique For Stability Condition}

The diffusion equation (2) and in general any parabolic partial differential equation has the first derivative in time and the finite difference, in general, uses the first-order backward, or forward, finite difference scheme which it two levels to deal with stability by Von Neumann stability technique.

More than two-time level schemes are rarely used but the timefractional finite difference scheme will be $n$ - level scheme (multi-level) due to memory and hereditary properties of the fractional-order derivative. and so the time-fractional finite difference scheme may be considered as multi-level schemes and we will use the techniques of multi-levels to find the stability conditions. for more details see (Adel Rashed 2016, Mitchal 1980, Richtmyer 1967).

Assume we use a fractional-order derivative for the fractional time in equation (2), then we get a multilevel scheme in time because of the memory and hereditary properties of the fractional-order derivative, then equation (2) can be written as

$T^{n+1}=A_{n} T^{n}+A_{n-1} T^{n-1}+\cdots+A_{0} T^{0}$

where $A_{j}, j=0,1, \cdots, n$, are bounded linear operators. Enter a new alternating dependent variables $T^{n-1}$ by $U^{n}, T^{n-2}$ by $V^{n}$, $\cdots, T^{1}$ by $W^{n}$ and $T^{0}$ by $S^{n}$, then we can write (10) by the following system

$$
\begin{array}{ll}
T^{n+1} & =A_{n} T^{n}+A_{n-1} U^{n}+A_{n-2} V^{n}+\cdots \\
U^{n+1} & =T^{n} \\
V^{n+1} & =U^{n} \\
\vdots & \\
S^{n+1} & =W^{n}
\end{array}
$$

or

$\left[\begin{array}{l}T^{n+1} \\ U^{n+1} \\ \vdots \\ S^{n+1}\end{array}\right]=\left[\begin{array}{lllll}A_{n} & A_{n-1} & \ldots & A_{1} & A_{0} \\ I & O & \ldots & O & O \\ \vdots & \vdots & \ldots & \vdots & \vdots \\ O & O & \ldots & I & O\end{array}\right]\left[\begin{array}{l}T^{n} \\ U^{n} \\ \vdots \\ S^{n}\end{array}\right]$

Define $\boldsymbol{\Psi}^{\mathbf{n}+\mathbf{1}}=\left[T^{n+1}, U^{n+1}, \ldots, S^{n+1}\right]^{T}, \quad \boldsymbol{\Psi}^{\mathbf{n}}=$

$\left[T^{n}, U^{n}, \ldots, S^{n}\right]^{T}$ and the multilevel amplification matrix $A$ as a 
block square matrix of order $(n+1)$ :

$\mathbf{A}=\left[\begin{array}{lllll}A_{n} & A_{n-1} & \ldots & A_{1} & A_{0} \\ I & 0 & \ldots & 0 & 0 \\ \vdots & \vdots & \ldots & \vdots & \vdots \\ 0 & 0 & \ldots & I & O\end{array}\right]$

for simplicity suppose $M=N$, then every element of $A$ is a square matrix of order $(M-1) \times(M-1)$, with $I, O$ denote the identity and zero operators, and $[\mathrm{A}]^{T}$ denote the transpose, then equation (10) can be written as a system of $[(M-1) \times$ $(M-1) \times(n+1)]$ equations

$$
\boldsymbol{\Psi}^{n+1}=\mathbf{A} \boldsymbol{\Psi}^{n}
$$

Introduce a exemplary Fourier term $\boldsymbol{\Phi}_{l, m}^{n}=\boldsymbol{\Phi}_{0,0}^{n} e^{i \beta l h+i \gamma m k}$, where $\Psi_{0,0}^{n}$ is a constant matrix, substitute this in (14) to get

$$
\Psi_{l, m}^{n+1}=M \Psi_{l, m}^{n}
$$

where $M$ will be the amplification matrix, we have Now a two-level problem $n$ and $n+1$. The stability condition can be evaluated from the eigenvalues of the amplification matrix $M$, i.e. the maximum absolute eigenvalue must be less or equal to one (Richtmyer 1967).

\section{Stability Conditions}

We will use the multilevel technique to find the stability conditions of the explicit finite difference scheme.

Theorem 1: The fractional explicit scheme (9) is conditionally stable and the stability condition is $r_{\alpha} \leq$ $\frac{1-2^{-\alpha}}{2 \Gamma(2-\alpha)}$.

Proof. The scheme (9) is a multilevel scheme and can be rewritten as

$$
\begin{aligned}
T_{i, j}^{n+1}=\left[c_{1}^{\alpha}+\right. & \left.r_{\alpha} \Gamma(2-\alpha)\left(\delta_{x}^{2}+\delta_{y}^{2}\right)\right] T_{i, j}^{n} \\
& +\sum_{k=2}^{n} c_{k}^{\alpha} T_{i, j}^{n-k+1}+b_{n}^{\alpha} T_{i, j}^{0}+\frac{1}{w_{\alpha}^{\tau}} Q_{i, j}^{n}
\end{aligned}
$$

then the multilevel amplification matrix $A$ can be defined by square block matrix of order $(n+1)$ and every element of $A$ is of order $(J-1) \times(J-1)$ :

$$
\begin{aligned}
& \mathbf{A}= \\
& {\left[\begin{array}{llllll}
{\left[c_{1}^{\alpha}+r_{\alpha} \Gamma(2-\alpha)\left(\delta_{x}^{2}+\delta_{y}^{2}\right)\right] I} & \left(c_{2}^{\alpha}\right) I & \left(c_{3}^{\alpha}\right) I & \ldots & \left(c_{n}^{\alpha}\right) I & \left(b_{n}^{\alpha}\right) I \\
I & O & O & \ldots & O & 0 \\
O & I & O & \ldots & 0 & 0 \\
\vdots & \vdots & \vdots & \ldots & O & \vdots \\
O & O & O & \ldots & I & 0
\end{array}\right]}
\end{aligned}
$$

for the amplification matrix Insert expression

$T_{i, j}^{n}=\xi^{n} e^{m \beta i h} e^{m \gamma j k}$

where $m=\sqrt{-1}, \beta, \gamma$ are real spatial wave numbers and $\xi$ is the amplification factor.

$$
\begin{aligned}
T_{i, j}^{n+1} & =b_{n}^{\alpha} T_{i, j}^{0}+\sum_{k=1}^{n} c_{k}^{\alpha} T_{i, j}^{n-k+1} \\
& +r_{\alpha} \Gamma(2-\alpha)\left[\left(\delta_{x}^{2}+\delta_{y}^{2}\right) T_{i, j}^{n}\right]+\frac{1}{w_{\alpha}^{\tau}} Q_{i, j}^{n}
\end{aligned}
$$

This can be rewritten as

$$
\begin{aligned}
T_{i, j}^{n+1}= & b_{n}^{\alpha} T_{i, j}^{0}+\sum_{k=1}^{n} c_{k}^{\alpha} T_{i, j}^{n-k+1} \\
& +r_{\alpha} \Gamma(2-\alpha) \delta_{x}^{2} T_{i, j}^{n}
\end{aligned}
$$

$$
+r_{\alpha} \Gamma(2-\alpha) \delta_{y}^{2} T_{i, j}^{n}+\frac{1}{w_{\alpha}^{\tau}} Q_{i, j}^{n}
$$

Insert the expression in equation (18) to get:

$$
\begin{aligned}
& \xi^{n+1} e^{m \beta i h} e^{m \gamma j k}=b_{n}^{\alpha} \xi^{0} e^{m \beta i h} e^{m \gamma j k} \\
& \quad+\sum_{k=1}^{n} c_{k}^{\alpha} \xi^{n-k+1} e^{m \beta i h} e^{m \gamma j k} \\
& +r_{\alpha} \Gamma(2-\alpha) \xi^{n} e^{m \gamma j k}\left(e^{m \beta(i+1) h}-2 e^{m \beta i h}+e^{m \beta(i-1) h}\right) \\
& +r_{\alpha} \Gamma(2-\alpha) \xi^{n} e^{m \beta i h}\left(e^{m \gamma(j+1) k}-2 e^{m \gamma j k}+e^{m \gamma(j-1) k}\right)
\end{aligned}
$$

Divided by $e^{m \beta i n} e^{m \gamma j k}$ to get

$$
\begin{aligned}
\xi^{n+1} & =b_{n}^{\alpha} \xi^{0}+\sum_{k=1}^{n} c_{k}^{\alpha} \xi^{n-k+1} \\
& +r_{\alpha} \Gamma(2-\alpha) \xi^{n}\left(e^{m \beta h}-2+e^{-m \beta h}\right) \\
& +r_{\alpha} \Gamma(2-\alpha) \xi^{n}\left(e^{m \gamma k}-2+e^{-m \gamma k}\right)
\end{aligned}
$$

By using the formula $\left(e^{m \theta}-2+e^{-m \theta}\right)=-4 \sin ^{2} \frac{\theta}{2}$ we can get:

$$
\begin{aligned}
\xi^{n+1}= & b_{n}^{\alpha} \xi^{0}+\sum_{k=1}^{n} c_{k}^{\alpha} \xi^{n-k+1} \\
& +r_{\alpha} \Gamma(2-\alpha) \xi^{n}\left(-4 \sin ^{2} \frac{\beta h}{2}\right) \\
& +r_{\alpha} \Gamma(2-\alpha) \xi^{n}\left(-4 \sin ^{2} \frac{\gamma k}{2}\right)
\end{aligned}
$$

can be rewritten

$$
\begin{aligned}
& \xi^{n+1}=\xi^{n}\left[c_{1}^{\alpha}-4 r_{\alpha} \Gamma(2-\alpha)\left(\sin ^{2} \frac{\beta h}{2}+\sin ^{2} \frac{\gamma k}{2}\right)\right] \\
& +\sum_{k=2}^{n} c_{k}^{\alpha} \xi^{n+1-k}+\xi^{0} b_{n}^{\alpha}
\end{aligned}
$$

then the amplification matrix $M$ can be defined by square block matrix of order $(n+1)$ and every element of $M$ is of order $(J-1)$ :

$M=\left[\begin{array}{llllll}\left(c_{1}^{\alpha}-4 r_{\alpha} \Gamma(2-\alpha) \eta\right) I & \left(c_{2}^{\alpha}\right) I & \left(c_{3}^{\alpha}\right) I & \ldots & \left(c_{n}^{\alpha}\right) I & \left(b_{n}^{\alpha}\right) I \\ I & O & O & \ldots & O & O \\ O & I & O & \ldots & O & O \\ \vdots & \vdots & \vdots & \ldots & \vdots & \vdots \\ O & O & O & \ldots & I & O\end{array}\right]$

where $\quad \eta=\sin ^{2} \frac{\beta h}{2}+\sin ^{2} \frac{\gamma k}{2} \quad$ and $\quad 0 \leq \eta \leq 2$. From Gerschgorin theorem for estimating the eigen values of any matrix (Varga 2004), all rows of matrix $M$ gives eigne values lies in the union of unit discs centered at $(0,0)$ in the complex plan except those corresponding to the first block. For each row of the first block one can see that the corresponding eigenvalue satisfies

$|\lambda| \leq\left|c_{1}^{\alpha}-4 r_{\alpha} \Gamma(2-\alpha) \eta\right|+\sum_{k=2}^{n}\left|c_{k}^{\alpha}\right|+\left|b_{n}^{\alpha}\right|$

from [8] (the properties of $c_{k}^{\alpha}$ ) we have $c_{k}^{\alpha}>0, b_{n}^{\alpha}>0$, and $\sum_{k=2}^{n} c_{k}^{\alpha}=2^{1-\alpha}-1-b_{n}^{\alpha}$, this lead to

$|\lambda| \leq\left|2-2^{1-\alpha}-4 r_{\alpha} \Gamma(2-\alpha) \eta\right|+2^{1-\alpha}-1$

if the right hand inequality is less than or equal to one then $|\lambda| \leq 1$, then we have

$\left|2-2^{1-\alpha}-4 r_{\alpha} \Gamma(2-\alpha) \eta\right|+2^{1-\alpha}-1 \leq 1$

Or

$\left|2-2^{1-\alpha}-4 r_{\alpha} \Gamma(2-\alpha) \eta\right| \leq 2-2^{1-\alpha}$

then

$-\left(2-2^{1-\alpha}\right) \leq\left(2-2^{1-\alpha}-4 r_{\alpha} \Gamma(2-\alpha) \eta\right) \leq 2-2^{1-\alpha}$ 
the right hand inequality is satisfied and we need to calculate the condition on $r_{\alpha}$ to make the left hand inequality satisfied, this lead to

$4 r_{\alpha} \Gamma(2-\alpha) \eta \leq 4-2^{2-\alpha}$

then the stability condition is

$r_{\alpha} \leq \frac{4-2^{2-\alpha}}{4 \eta \Gamma(2-\alpha)}=\frac{1-2^{-\alpha}}{\eta \Gamma(2-\alpha)} \leq \frac{1-2^{-\alpha}}{2 \Gamma(2-\alpha)}$

And this is the stability condition.

\section{Convergence}

To prove the convergence of the finite difference scheme (9), it is easy to prove the consistency and by theorem 1 we found the stability conditions, then the Lax equivalence theorem will guarantee the convergence of the scheme (9) (Richtmyer 1967).

\section{Numerical Example}

Example: Consider the two dimensional time fractional diffusion Equation (2) where $Q(x, y, t)=\left(e^{t} \frac{\partial^{\alpha}}{\partial t^{\alpha}} e^{-t}+\right.$ $\left.2 \pi^{2}\right) T(x, y, t)$ with initial condition $T(x, y, 0)=$ $\sin (\pi x) \sin (\pi y)$ and Dirichlet boundary condition $T(0, y, t)=T(1, y, t)=T(x, 0, t)=T(x, 1, t)=0$. The exact solution is $T(x, y, t)=e^{-t} \sin (\pi x) \sin (\pi y)$.

In the calculation, we consider two estimators of error, the Maximum Error, and the Average Error. We define the Maximum Error as $\left\{\max \left|T_{i, j}^{k}-T\left(x_{i}, y_{j}, t_{k}\right)\right| ; 0<i<M, 0<\right.$ $j<N\}$ and the Average Error define as $\left\{\frac{\sum_{i=1}^{M-1} \sum_{j=1}^{N-1}\left|T_{i, j}^{k}-T\left(x_{i}, y_{j}, t_{k}\right)\right|}{(N-1)(M-1)}\right\}$. In the calculations, we considered two different values of $\alpha$ as shown in table 1.

\begin{tabular}{||l|l|l|l|l||}
\hline \hline$\alpha$ & $\Delta t$ & $\Delta x=\Delta y$ & Average Error $\times 10^{-3}$ & Maximum Error $\times 10^{-3}$ \\
\hline \hline 0.8 & 0.005 & 0.25 & 0.203427670508 & 2.048555466561 \\
\hline 0.8 & 0.00075 & 0.125 & 0.127161039579 & 0.937551386860 \\
\hline 0.8 & 0.0005 & 0.1 & 0.104228938934 & 0.705978327718 \\
\hline 0.8 & 0.000075 & 0.05 & 0.058822452859 & 0.251585258180 \\
\hline 0.6 & 0.0005 & 0.25 & 0.179065904487 & 1.783584536434 \\
\hline 0.6 & 0.000005 & 0.125 & 0.128874412078 & 0.865696744094 \\
\hline 0.6 & 0.00002 & 0.1 & 0.136206891713 & 0.695969744479 \\
\hline 0.6 & 0.0000025 & 0.05 & 0.102045906207 & 0.343680626193 \\
\hline 0.5 & 0.0002 & 0.25 & 0.162267719795 & 1.159274142619 \\
\hline 0.5 & 0.00001 & 0.125 & 0.135508996908 & 0.813420658726 \\
\hline 0.5 & 0.000005 & 0.1 & 0.113616405293 & 0.707983555430 \\
\hline 0.5 & 0.0000034 & 0.05 & 0.100336833230 & 0.383275082559 \\
\hline
\end{tabular}

Table 1: The Average Error and maximum error at $t=0.6$ with various values of $\Delta x$

\section{Conclusion}

The explicit finite difference scheme is conditionally stable, the solution can be obtained easily but restrict the time step and this is mean more computational work. To find the stability conditions we using the classical multilevel schemes techniques with Gerschgorin's discs theorems and this method is Successful and the stability condition depends on the order of fractional derivative $\alpha$. we tested the stability conditions on a numerical example and found a good agreement with the theoretical results.

Acknowledgments: The authors would like to thank the anonymous referees and Editor-in-Chief for reading this work.

\section{References}

[1] Adel Rashed Abd Ali Alsabbagh and Ismail Kaoud Youssef. Numerical Treatment Based on Spectral Methods for Diffusion Like Problems. PhD thesis, Ain Shams University. Faculty of Science.
Department of mathematics, thesis(Ph.D), 2016.

[2] R. Herrmann. Fractional calculus: An introduction for physicists. World Scientific, 2011.

[3] K.S. Miller and B. Ross. An Introduction to the Fractional Calculus and Fractional Differential Equations. New York : Wiley-Interscience Publ., 1993.

[4] A. R. Mitchal and D. F. Griffiths. The Finite Difference Method in Partial Differential Equations. John Wiley and Sons, 1980.

[5] I. Podlubny. Fractional Differential Equations. Camb. Academic Press, San Diego, CA, 1999.

[6] Robert D. Richtmyer and K. W. Morton. Difference Methods for Initial Value Problems. Interscience publishers, second edition, 1967. 
[7] Richard S. Varga. Gersgorin and His Circles, volume 36 of Springer Series in Computational Mathematics. Springer-Verlag Berlin Heidelberg, first edition, 2004.

[8] P. Zhuang and F. Liu. Finite difference approximation for two-dimensional time fractional diffusion equation. Journal of Algorithms \& Computational Technology, 1(1):1-16, 2007. 